\title{
Género, salud mental y terapia ocupacional: algunas reflexiones sobre la influencia de la historia de las mujeres y la perspectiva de género en nuestras prácticas*
}

\section{Gender, mental health and occupational therapy: some reflections on the influence of the history of women and the gender perspective in our practice}

\author{
Daniela E. Testa ${ }^{1}$, Sandra B. Spampinato ${ }^{2}$
}

\begin{abstract}
TESTA, D. E.; SPAMPINATO, S. B. Género, salud mental y terapia ocupacional: algunas reflexiones sobre la influencia de la historia de las mujeres y la perspectiva de género en nuestras prácticas. Rev. Ter. Ocup. Univ. São Paulo, v. 21, n. 2, p. 174-181, maio/ago. 2010.

RESUMEN: Se presentan algunas reflexiones sobre la problemática de género en las prácticas de salud mental y de Terapia Ocupacional a partir de dos situaciones históricas que muestran, por un lado aspectos vinculados con la actuación de las mujeres en el ámbito público, y por otro destacan la implicancia de las relaciones de género en dichas actuaciones. El objetivo es analizar el impacto de las representaciones de género en el campo de la salud mental y su relación con las prácticas actuales de la Terapia Ocupacional. Se presentan los elementos constitutivos del concepto de género para identificar las lógicas asistenciales destinadas a dar respuesta a la problemática de salud mental, que reproducen el entramado de poder y dominación característico de las relaciones de género. Se propone incluir la perspectiva de género en las prácticas de la Terapia Ocupacional cada vez que se instrumenten intervenciones, estrategias o dispositivos en salud; teniendo presente que la tensión entre la práctica de la Terapia Ocupacional y los condicionamientos del sistema de género, está siempre en relación al proceso particular de subjetivación de cada uno de los sujetos con los que se interviene.
\end{abstract}

DESCRITORES: Terapia ocupacional; Prática profissional; Saúde mental; Gênero e saúde; Mulheres/educação; Trabalho feminino/história.

\footnotetext{
* Este artículo está basado en el trabajo "Implicancia de la perspectiva de género en las prácticas de Salud Mental y Terapia Ocupacional" presentado en el $15^{\circ}$ Congreso Mundial de la Federación Mundial de Terapia Ocupacional "Ocupación desde Latinoamérica" Chile, 4 al 7 de Mayo de 2010.

1. Licenciada en Terapia Ocupacional. Maestranda en "Diseño y Gestión de Políticas Públicas" - FLACSO Argentina. Coordinadora Emprendimiento Social en Salud - Servicio de Terapia Ocupacional - Hospital "José T. Borda” - Buenos Aires - Argentina. Docente de la Universidad Nacional de San Martín- Universidad Nacional de Buenos Aires.

2. Licenciada en Terapia Ocupacional. Licenciada en Terapia Ocupacional. Maestranda en "Diseño y Gestión de Políticas Públicas" FLACSO Argentina. Coordinadora Emprendimiento Social en Salud - Servicio de Terapia Ocupacional - Hospital "José T. Borda” Buenos Aires - Argentina. Docente de la Universidad Nacional de San Martín- Universidad Nacional de Buenos Aires.

Endereço para correspondencia: danitestu@yahoo.com.ar / sandraspampinato@yahoo.com.ar
} 
$\mathrm{P}$ ara abordar la problemática de la perspectiva de género en las prácticas de Salud Mental y específicamente en Terapia Ocupacional, vamos a valernos de dos situaciones históricas que representan momentos fundacionales donde las mujeres asumen un lugar protagónico.

La primera situación se refiere a la definición de la locura como un problema de interés del Estado. La segunda referencia trata sobre la creación de la carrera de Terapia Ocupacional en Argentina.

Sin la presunción de dar cuenta de un proceso histórico con la complejidad que ello requeriría, encararemos el presente artículo a través de una revisión bibliográfica para analizar posibles relaciones con las prácticas de Terapia Ocupacional actuales.

Luego, mencionaremos algunos aspectos conceptuales de la perspectiva de género, para, por último, compartir algunas reflexiones referidas a la Terapia Ocupacional.

Para ello tomaremos aportes historiográficos acerca de la política manicomial en la ciudad de Buenos Aires en el período 1880-1940 (DE BAYÓN, 2000; STAGNARO, 2006) y sobre la historia de las mujeres en Argentina (GIL LOZANO et al., 2000; BARRANCOS, 2007; PITA, 2009).

\section{El problema de "las dementes"}

En el marco del proceso de construcción del Estado Nación Argentino -1816-, la definición de la locura como un problema y su instalación en la agenda pública, se debió al diseño y las negociaciones de un grupo de mujeres, que asumieron un compromiso ante la preocupación de la época por lograr el ideal de "orden y el progreso" en una realidad fragmentada por las luchas externas e internas dadas en el país (SPAMPINATO, 2007).

En 1823, más de 40 mujeres de la elite de la sociedad de Buenos Aires conformaron la Sociedad de Beneficencia. Integrada al organigrama estatal desde sus inicios, y financiada principalmente con recursos del Estado, se convirtió en una plataforma para ejercer la participación e influenciar en la arena pública: ocuparon cargos jerárquicos en ella, administraron recursos y presupuestos de manera autónoma, manejaron la prensa.

Estas damas, interesadas por la política, no cuestionaron los límites de una ciudadanía formal que les permitiera votar, sino que ejercieron un status ciudadano a través de la "maternidad civil y social" basado en las virtudes del sexo femenino y una posición social privilegiada (PITA, 2009).
Mediante los diferentes programas de tutela fueron dando densidad al ordenamiento estatal, a la vez que consolidaron una esfera de acción pública a partir de la cual influenciaron en los debates -de la época- sobre la formación de la Nación y la civilidad.

En principio se ocuparon de las mujeres y los niños pobres, y a partir del año 1852 ampliaron sus incumbencias a la atención hospitalaria (DI STÉFANO et al., 2002; GOLBERG, 2004).

Es en este momento en el que instalan y definen el problema de "las locas" en la agenda pública, marcando el curso de acción y - en consecuencia - iniciando todas las gestiones necesarias para la creación del "Hospital de Mujeres Dementes", que finalmente se funda entrado el año 1854.

Estas mujeres definieron quiénes y cómo las mujeres debían ser incluidas en el proyecto de Nación, en tal sentido la figura de "la demente", según expresiones de la época, funcionó para demarcar el límite entre la inclusión y la exclusión a la vida pública, a la vez que legitimaba su función de tutoras civiles y sociales. Así la tutela pública de la locura se convirtió en un asunto de mujeres.

Las locas eran vistas como las "afligidas" o "pobres e infelices mujeres", aunque se destacaba su laboriosidad. Esta visión determinó el modelo de intervención "pues, aunque faltas de razón hacen muy buen uso de sus manos y cosen bastante bien", se lee en los relatos de la época (ACTAS SOCIEDAD BENEFICENCIA, 1823).

En relación a las representaciones de género la figura de la mujer estaba asociada a la virtud, la crianza de los hijos, el buen desempeño de la administración del hogar y la preservación de los valores morales.

Con el transcurso del tiempo se convirtió en la institución paradigmática de la beneficencia, administrando muchas de las primeras instituciones que conformaron luego el Sistema de Salud Argentino y consolidó el modelo asistencial de la Caridad Pública, que ha marcado fuertemente las políticas sociales de nuestro país.

\section{Epidemia de Polio y Carrera de Terapia Ocupacional}

Durante el año 1956, en el marco de una de las más graves epidemias de poliomielitis en la Argentina, un grupo de mujeres inicia los cursos de Terapia Ocupacional que luego darán origen a la carrera; asumiendo un compromiso frente al amenazado ideal de progreso y crecimiento nacional que esta enfermedad representaba.

La magnitud de esta epidemia cuestionó las respuestas y la capacidad resolutiva del estado y de 
TESTA, D. E.; SPAMPINATO, S. B. Género, salud mental. Rev. Ter. Ocup. Univ. São Paulo, v. 21, n. 2, p. 174-181, maio/ago. 2010.

las organizaciones de salud, marcando el inicio de la reconversión de las prácticas sanitarias que daban asistencia a las víctimas de la enfermedad, en su mayoría niños. La epidemia aconteció en medio de una crisis política e institucional más amplia unos meses después del golpe de estado que derrocó al primer gobierno peronista a fines de 1955. (TESTA, 2008).

En un contexto de crecimiento y expansión, la presencia de parte de una generación afectada por las secuelas motoras de la poliomielitis, representaba un problema económico, dado que restaba fuerza de trabajo al mercado, y en el mejor de los casos, luego de una buena recuperación, disminuía su rendimiento (RAMACCIOTTI, 2009).

La creación de la carrera de T.O fue una de las acciones implementadas por el Estado para enfrentar el problema de la rehabilitación y la reinserción social, plena y útil de los niños afectados por la polio. Se creó como una carrera complementaria de la medicina de avanzada de la época, a partir de una falta de respuesta por parte de los saberes hegemónicos.

De acuerdo a las tendencias del momento, la Terapia Ocupacional formaba parte de un equipo de variados profesionales y técnicos coordinados por médicos, todos dedicados a la rehabilitación del aparato locomotor (MACDONALD, 1959; GONZALEZ, 1959).

Queremos destacar que la Terapia Ocupacional en Argentina nació a partir de una situación de emergencia sanitaria y altos grados de incertidumbre, en un momento en que no había respuestas garantizadas sobre cómo tratar exitosamente las secuelas de la poliomielitis. Este fue el contexto sobre el que se conformó el naciente campo de la Terapia Ocupacional en el país, profesión que aceptó el desafío de crear respuestas "no inventadas" e intervenir en medio de la crisis sanitaria.

Hemos considerado ambas referencias históricas para pensar cómo las relaciones de género, y la figura de la mujer en particular, fueron determinantes a la hora de recomponer relaciones sociales fragmentadas en el mundo público, específicamente -al menos en estos casos en la Argentina- en momentos históricos en donde lo que se vio amenazado fue el imaginario del orden y el progreso de la sociedad (PITA, 2009).

\section{Algunos Lineamientos Conceptuales}

Para continuar, traemos a cuenta algunos lineamientos conceptuales básicos que hemos considerado para el análisis.

El género es un concepto construido socialmente, es la forma simbólica de la diferencia sexual que se funda culturalmente en un conjunto de prácticas, ideas y discursos. Abarca las pautas culturales de dominación, control y resistencia que moldean lo sexual y los discursos sociales que organizan los significados (FRASER, 2000; LAMAS, 2002).

El sistema de género hace referencia a los procesos y mecanismos sociales que regulan y organizan la sociedad de modo que mujeres y hombres sean, actúen y se consideren diferentes, al mismo tiempo que determina cuáles áreas sociales son competencia de un sexo y cuáles del otro (ASTELARRA, 2004).

El género por lo tanto no se asigna sólo a las personas sino también a las actividades mismas. Es así que históricamente se le ha adjudicado al hombre la vida pública y a la mujer la vida privada. Aunque uno no puede existir sin el otro, el mundo público no sería posible sin la infraestructura material generada en el mundo privado.

Hablar de diferencias de género implica poner en visibilidad las relaciones de poder entre mujeres y hombres y aceptar que el problema no radica en las especificidades anatómicas sino en las desigualaciones que las sociedades han construido a partir de las mismas. Alude indefectiblemente a los dispositivos de poder que han justificado las desigualdades a partir de las diferencias sexuales.

No aceptarlo así despolitiza el enfoque. Al perderse la dimensión política- es decir al invisibilizarse las consecuencias de las asimetrías de poder, se conforman los invisibles sociales, constituidos por hechos, dispositivos, acontecimientos y procesos que se reproducen social y subjetivamente.

\section{Implicancia para la Terapia Ocupacional}

Ahora bien; ¿Cómo pensar la implicancia de género y la Terapia Ocupacional? ¿Cómo pensar críticamente el tema, desde una profesión, elegida y ejercida predominantemente por mujeres?

Existen numerosos trabajos de colegas que han destacado la relación entre los modelos teórico prácticos elegidos (que siempre resultan de presupuestos filosóficos y científicos), el modo de intervención y la manera en que se aplican las actividades como recurso terapéutico, señalando que cada una de estas elecciones está condicionada por una finalidad política que se corresponde con un campo social específico, en el cual se inscriben las instituciones, las estrategias y la modalidad de las intervenciones (PAGANIZZI, 1997, 2001, 2005; CAPOZZO, 2007; MARTÍNEZ ANTÓN, 1998, 2000; 


\section{GALHEIGO, 2007).}

Como agentes de salud reconocemos que para hacer visible las relaciones de poder que también nos implican, para repensar las prácticas desde esta perspectiva, es necesario desprenderse de los modelos referenciales anteriores. Nos exige reflexionar sabiéndonos atravesados por configuraciones de poder que nos implican directamente en las dimensiones subjetiva, profesional y política (GALHEIGO, 2007).

En palabras de Medeiros (2008, p. 84) "no es sólo una cuestión de estilo", tiene que ver con replantear también dichos modelos así como los valores asumidos en lo cotidiano, tanto en las relaciones personales como profesionales.

Se trata de develar la naturaleza política de las ocupaciones y los espacios de cotidianeidad; de modo tal que no sean un mero instrumento de adaptación al medio, reconociendo que representan la posibilidad de influenciar en los ámbitos sociales dada la capacidad transformadora de la actividad humana.

En cuanto a la salud mental, Franco Basaglia (1972) destaca que se ha utilizado una ideología de la diferencia sobre la enfermedad mental que aseguró la marginación social de aquellos considerados como diferentes, adoptando sistemas de control social que garanticen tal marginación, de acuerdo con diversos niveles de desarrollo socioeconómico y las ideas claves de la clase dominante. Las mujeres han caído dentro de esta práctica de castigar lo diferente.

Nancy Fraser (2000) aporta reflexiones sobre el reconocimiento que nos ayudan a pensar sobre la situación de exclusión de las mujeres con padecimiento mental grave, debido a que destaca las luchas a favor del reconocimiento de las diferencias, que aspiraban a la reafirmación de la identidad así como también a un mejor posicionamiento en relación a la distribución de riqueza y poder.

Como vimos en el punto referido a la historia, las locas formaron parte de la "cuestión social" del siglo XIX, encarnando una amenaza a los ideales de orden y progreso. Ahora bien, en la actualidad también siguen presentes en la actual "nueva cuestión social" formando parte de la masa de excluidos, de los irrelevantes como productores e insignificantes como consumidores (ROSANVALLON, 1995).

En este nuevo escenario la crítica de Fraser nos alerta, por un lado, sobre el riesgo de perpetuar las desigualdades a partir de abandonar la preocupación por la redistribución y, por el otro, sobre el peligro de justificar violaciones a los derechos humanos, el patriarcado y el autoritarismo.
Este señalamiento entiende que el enfoque de género debe ser comprendido como un problema de redistribución y no sólo como un problema de reconocimiento; de lo contrario queda velada la situación de accesibilidad inequitativa a los recursos materiales y simbólicos.

El punto de vista mencionado implica una política que se proponga superar la subordinación y alcanzar la igualdad de status como miembro pleno de la sociedad. Inferiores, excluidos, invisibles, contenidos en una relación institucionalizada de subordinación social, equivale a "no ver reconocido el status de participante de pleno derecho en la interacción social como una consecuencia de los modelos institucionalizados que construyen a una persona como comparativamente indigna de respeto o estima." (FRASER, 2000)

La falta de reconocimiento va más allá de las representaciones y discursos; está perpetrada en los modelos culturales institucionalizados y en los funcionamientos que regulan la interacción social a través de normas y prácticas. Así es como se trata la situación de las mujeres con sus identidades reducidas a la de pacientes; por ejemplo a través de normas que regulan las relaciones con los hijos, el manejo del dinero, el ejercicio de los derechos civiles durante la internación psiquiátrica, etc, que suelen lesionar la igualdad y la equidad en el acceso a los derechos.

No nos queda más que afirmar que se evidencia que la lógica de género es lógica de poder y dominación, asumiendo la forma paradigmática de la violencia simbólica (BORDIEU, 1991) que se reproduce sin mayores cuestionamientos en las lógicas asistenciales destinadas a dar respuesta a la problemática de salud mental.

Lo cierto es que el prejuicio también oculta números cada vez más alarmantes de los padecimientos mentales femeninos: se estima que, en 2020, éstos serán la segunda causa de muerte y discapacidad de las mujeres. Pero aunque la realidad de la mujer hoy es bien distinta, algunas de las conductas parecen aún permanecer a través de las generaciones. Las cifras de la OMS -Organización Mundial de la Salud - revelan un panorama sombrío para las mujeres con respecto a los trastornos depresivos: los casos de discapacidad por trastornos neuropsiquiátricos llegan al 41,9 por ciento, cuando en los hombres llega apenas al 29,3 por ciento. La OMS también calcula que para 2020 los trastornos de este tipo serán la segunda causa de muerte y discapacidad a nivel mundial (la primera hoy son las cardiopatías) (MONTERO et al., 2003)

A partir del estudio de Montero et al. (2003) que demuestran que el incremento de la morbilidad psiquiátrica en mujeres casadas de mediana edad, en comparación 
con las solteras, es un hecho frecuentemente observado, y además que, en esencia, hoy se admite que dicha asociación es un rasgo característico de las mujeres, que no aparece en los hombres, para los que el estado civil casado se comporta como un factor de protección frente al desarrollo de la enfermedad mental, debemos necesariamente replantear la idea de familia que subyace a estos propósitos.

Estos autores también proponen reflexionar sobre el controvertido papel del trabajo en los procesos de rehabilitación o inserción social. El papel que el trabajo desempeña en el bienestar psicológico de las mujeres es complejo y depende de una amplia serie de variables ligadas a la naturaleza de éste, su valoración social y las posibilidades que ofrece en su proceso de desarrollo personal. La referencia a la situación laboral de la mujer en abstracto resulta hoy inadecuada. A la hora de analizar el papel que el trabajo puede ejercer en la salud mental de la mujer, es necesario tener en cuenta los aspectos inherentes al significado y el sentido personal y social del mismo (MONTERO et al., 2003).

Estos cambios indudablemente afectan tanto a los hombres como a las mujeres, pero plantean una problemática, que resulta particular a las mujeres, sobre todo a las casadas con hijos pequeños pertenecientes a las clases sociales más desfavorecidas, lo cual se agudiza en las mujeres con padecimiento mental, mayoritariamente pobres que en se asisten en el sistema público de salud.

\section{CONCLUSIONES}

Para terminar, queremos plantear la conclusión en torno a dos ejes:

- el primero focaliza en las referencias históricas, considerando que el hecho histórico también habla del presente y nos es útil para entenderlo y contextualizarlo.

- el segundo plantea una mirada transversal a los procesos de salud en la práctica de la Terapia Ocupacional.

En cuanto a las Referencias Históricas, podemos decir que:

En el caso de la Sociedad de Beneficencia, las condiciones materiales no determinaron por sí solas los procesos de subjetivación, pero sí condicionaron las formas en que las lógicas de género se hicieron presentes en los distintos ámbitos sociales. Como vimos, las mujeres de la elite porteña no tenían derecho al voto, pero debido a su posicionamiento social, obtenido por herencia y/o matrimonio, ejercieron ciudadanía a través de la maternidad social. Las mujeres de clases desfavorecidas ejercían una ciudadanía cercenada al lugar de tuteladas de las otras mujeres, que definían quiénes serían las merecedoras de su protección, en tanto virtuosas y laboriosas demostraran ser.

En el caso de la creación de la carrera de Terapia Ocupacional, estuvo atravesada por un sistema de género que estableció jerarquías, privilegios y desigualdades; delimitando un campo profesional ligado a un mundo considerado como naturalmente femenino constituido por las actividades de la vida diaria, las actividades artesanales y manuales, el cuidado de los niños y una cuota de amor y abnegación para llevar adelante el desafío de la rehabilitación.

Aunque bajo la dirección de los médicos, profesión ejercida en aquella época mayoritariamente por hombres, fue un grupo de mujeres las que delinearon el perfil de la carrera y dejaron su huella en la formación de los terapistas ocupacionales de Argentina. Si bien no desafiaron las estructuras de poder, iniciaron un camino dentro del panorama de incertidumbre y urgencia que planteaba la epidemia, demostrando la relevancia de sus acciones en el plano político concreto, en tanto respuesta impulsada por el Estado y ejecutada eficazmente por actores femeninos.

Estos ejemplos ilustran como las condiciones políticas y materiales dadas no determinan por sí solas las relaciones de género, también influyen los procesos de subjetivación que se enmarcan en condiciones sociales e históricas particulares. Si entendemos por género a las construcciones histórico-sociales que delimitan lo femenino y lo masculino, y que también son parte del lenguaje que antecede a la conformación de los sujetos de una cultura, no es posible dejar de considerar las articulaciones entre la subjetividad, lo social, las condiciones objetivas de existencia y las experiencias. Las prácticas generadas en los diferentes espacios sociales ponen en juego las estrategias a través de las cuales los sujetos se apropian de los recursos simbólicos, que sólo se puede producir en el espacio intersubjetivo (SAUTÚ, 2001; FERNANDEZ, 2009).

En cuanto a la Mirada Transversal a la Práctica de la Terapia Ocupacional, destacamos los siguientes aspectos:

Las personas con sufrimiento mental constituyen un grupo que sufre desventajas sociales, culturales y económicas, siendo no menos "prisioneros de los mandatos de género" que el más común de los mortales de cualquier sexo.

Pareciera que la enfermedad mental se constituye 
en la identidad que vela la dimensión de género en el plano de la salud mental. Las categorías que aluden al status de diferente o anormal, legitiman la desigualdad y diluyen los efectos de las relaciones de poder jugadas en el sentido de lo femenino y lo masculino. Ser loco o loca, y pobre, ya resulta lo suficientemente opresivo, ¿para qué agregar la cuestión de género?

¿Cuánto de ello incluimos los terapistas ocupacionales en el análisis de las ocupaciones y/o actividades a la hora de instrumentar dispositivos y decidir estrategias terapéuticas?

En los procesos de salud las ocupaciones tienen una función primordial en tanto habilitan la entrada en un determinado orden cultural (MARTÍNEZ ANTÓN, 2000); aunque debido a su naturaleza compleja, también ponen de manifiesto la tensión entre lo social, lo comunitario y lo individual, que se juegan dentro de un determinado sistema de género.

Entonces, las ocupaciones y lo cotidiano tanto pueden ser el medio invisible eficaz y sutil para reproducir las relaciones establecidas, como el medio para desafiar los límites de lo considerado posible e incrementar y potenciar las libertades.

Pero este desafío nos involucra desde nuestra función profesional en la responsabilidad de garantizar espacios donde sea factible desnaturalizar primero y cuestionar después las lógicas binarias instituidas. Proponemos incluir la perspectiva de género en las prácticas de la Terapia Ocupacional cada vez que se instrumenten intervenciones, estrategias o dispositivos en salud.

Sin olvidar que la tensión entre la práctica de la Terapia Ocupacional y los condicionamientos del sistema de género, está siempre en relación al proceso particular de subjetivación de cada uno de los sujetos con los que trabajamos.

En primer lugar, es necesario reconocer que la perspectiva de género representa siempre un conflicto que refleja intereses y expresa relaciones de poder. Requiere identificación de oportunidades y resistencias, tanto en los productos y prácticas visibles de las organizaciones como en los valores y motivaciones no explícitos que determinan y dan forma a los productos de una organización. Es necesario hacer énfasis en la interrelación de los componentes ideológicos, entiéndase en los paradigmas, supuestos y enfoques teóricos.

Luego, sostenemos que las reivindicaciones pasan por desinstitucionalizar los modelos de valor cultural, por la transformación de las instituciones y de los valores. Cómo se realice dependerá en cada caso del modo en que la falta de reconocimiento se haya institucionalizado (FRASER, 2000).

En el caso de las prácticas de Terapia Ocupacional se trataría de examinar con atención los modos de asistencia y los tipos de dispositivos, las relaciones entre los asistidos, la identificación de sus necesidades y el acceso al ejercicio de sus derechos. Se destaca la contradicción entre la concepción lineal de la cura- rehabilitación obtenida a partir de la revinculación socio-laboral - que resulta un gran desafío en tiempos actuales - y la posibilidad única de obtener protección del Estado a partir de calificar para recibir asistencia a partir de la acreditación de la invalidez.

Si el autoempoderamiento se ve como un factor clave en la equidad de género, un primer paso debe ser el aprovisionamiento eficaz de espacios, recursos y tiempo que les permitan a las mujeres articular sus propios intereses en vez de que otros los prevean y satisfagan en su nombre. Los planificadores pueden ser los principales aliados pero los actores principales deben ser aquellos cuyas voces han sido acalladas y reprimidas.

Hay un largo camino por delante para lograr transformaciones en el campo de la salud mental donde, por supuesto, las relaciones de género están entretejidas en un contexto más amplio de relaciones sociales de poder hegemónicamente masculinas que estructuran la división de los recursos, responsabilidades, exigencias y obligaciones.

Los recursos intangibles, como la conciencia colectiva, la construcción de solidaridad de grupo y las habilidades de organización, son cruciales para la participación en la comunidad. Mejorar las capacidades de negociación y las habilidades para ejercer control sobre la propia vida, deberían ser preocupación central en una Terapia Ocupacional preocupada por incluir el enfoque de género en sus prácticas.

Se trata también de discutir el costado meramente productivista y moralizador del trabajo y la familia; ambos aspectos ampliamente incorporados y naturalizados en los procesos terapéuticos, en nuestra forma de pensar, sentir y actuar como profesionales y como pacientes.

Se hace necesario, entonces, identificar la distribución de las relaciones de poder y de los recursos materiales y simbólicos, que dan sentido y refuerzan los roles de género que se hallan presentes en la estructura y organización de los servicios sanitarios, con el fin de hacer hincapié en la construcción de otro posible sentido y en la producción de valor social viable a partir del respeto de las diferencias. 
TESTA, D. E.; SPAMPINATO, S. B. Género, salud mental. Rev. Ter. Ocup. Univ. São Paulo, v. 21, n. 2, p. 174-181, maio/ago. 2010.

TESTA, D. E.; SPAMPINATO, S. B. Gender, mental health and occupational therapy: some reflections on the influence of the history of women and the gender perspective in our practice. Rev. Ter. Ocup. Univ. São Paulo, v. 21, n. 2, p. 174-181, maio/ago. 2010.

\begin{abstract}
This work presents some reflections on the issue of gender in mental health and occupational therapy practices of two historical situations showing two different but related practices: on one hand aspects linked to the performance of women in the public domain and on the other hand, stand out the implication of gender relations in such action. This paper intent to analyze the impact of those representations of gender in the field of mental health and its relationship with the current practices of occupational therapy. It presents the constituent elements of the concept of gender to identify care logic aimed at responding to the issue of mental health, reproducing the structure of power and domination of gender relations. Intents to include gender mainstreaming in occupational therapy practices wherever instrument interventions, strategies, or devices in health, bearing in mind that the tension between the practice of occupational therapy and the gender system constraints, is always in relation with the particular process of subjectivation of each of the subjects with which intervenes.
\end{abstract}

KEY WORDS: Occupational therapy; Professional practice; Mental health; Gender and health; Women/history; Women, working/history.

\title{
REFERÊNCIAS
}

ACTA DE INSTALACIÓN DE LA SOCIEDAD DE BENEFICENCIA, 12 de abril de 1823. Instituciones de la Sociedad de Beneficencia y Asistencia Social (1823-1952), Documentos Escritos. Archivo General de la Nación. Tomo I.

ASTELARRA, J. Políticas de género en la Unión Europea y algunos apuntes sobre América Latina. Santiago de Chile: CEPAL, 2004. (Serie Mujer y Desarrollo N 57).

BARRANCOS, D. Mujeres en la sociedad argentina: una historia de cinco siglos. Buenos Aires: Ed. Sudamericana, 2007.

BASAGLIA, F. La institución negada. Ed. Barral. Barcelona: Ed. Barral, 1972.

BOURDIEU, P. El sentido práctico. Madrid: Ed. Taurus, 1991.

CAPOZZO, M. Aplicación de modelos teóricos en terapia ocupacional. [Mimeo, 2007].

DI STEFANO, R.; et al. De las cofradías a las organizaciones de la sociedad civil. Buenos Aires: Ed. Gadis, 2002.

FERNÁNDEZ, A. Las lógicas sexuales: amor, política y violencias. Buenos Aires: Ed. Nueva Visión, 2009.

FRASER, N. Nuevas reflexiones sobre el reconocimiento. New Left Rev., n. 4, Sept/Oct. 2000.

GALHEIGO, S. Terapia ocupacional en el ámbito social: aclarando conceptos e ideas. In: KRONENBERG, F.; SIMÓ-ALGADO, S.; POLLARD, N. Terapia ocupacional sin fronteras. Aprendiendo del espíritu de supervivientes. Buenos Aires: Ed. Médica
Panamericana, 2007. Cap. 7, p.85-87.

GIL LOZANO, F.; PITA, V.; INI, M. G. La historia de las mujeres en la Argentina. Buenos Aires: T 1. Ed. Taurus, 2000.

GOLBERG, L. Notas sobre la situación de la historiografía sobre la política social en la Argentina. In: BERTRANOU, J.; PALACIO, J. M.; SERRANO, G. (coord.). En el país del no me acuerdo. Buenos Aires: Ed. Prometeo, 2004.

GONZALEZ, L. Reunión inaugural. In: CONGRESO ARGENTINO DE REHABILITACIÓN DEL LISIADO, 3., 1959. Anales. $1^{\circ}$ parte. Mar del Plata, 1959. p.19-26.

LAMAS, M. Cuerpo: Diferencia sexual y género. Usos, dificultades y posibilidades de la categoría "género". Buenos Aires: Ed. Taurus, 2002.

MACDONALD, M. Conferencia la terapia ocupacional en la rehabilitación del Lisiado. In: CONGRESO ARGENTINO DE REHABILITACIÓN DEL LISIADO, 3., 1959. Anales. $2^{\circ}$ parte. Mar del Plata, 1959.

MARTÍNEZANTÓN MARTÍNEZ, R. La actividad y la ocupación. Rev. Ter. Ocup. Materia Prima, n. 14, p 9-12, 2000.

MARTIINEZ ANTÓN MARTÍNEZ, R. La terapia ocupacional. Historia y actualidad. La intervención. Apuntes materia: teoría y técnica de terapia ocupacional I. Carrera de terapia ocupacional. Universidad de Buenos Aires, 1998.

MEDEIROS, H. R. Terapia ocupacional. Un enfoque epistemológico y social. Argentina: Universidad Nacional del Litoral, 2008. 
MONTERO, I. APARICIO, D.; et al. Género y salud mental en un mundo cambiante. Sociedad Española de Epidemiología Psiquiátrica. Gaceta Sanitaria, v. 18, n. 1, 2004 (Ejemplar dedicado a: Informe SESPAS 2004: La salud pública desde la perspectiva de género y clase social).

PAGANIZZI, L. Actividad. Lenguaje particular. Buenos Aires: Edigraf, 1997.

PAGANIZZI, L. Actuaçao da terapia ocupacional no corpo marcado. In: CONGRESO BRASILEIRO DE TERAPIA OCUPACIONAL, 7., Octubre 2001. [Acesso em: mayo 2006]. Disponivel em: http://www.terapia-ocupacional.com

PAGANIZZI, L. Inserción social y trabajo informal. Experiencia y resultados. Avatares de la crisis, 2005. [Acesso em: mayo 2006] Disponível em: http://www.terapia-ocupacional.com

PITA, V. Género, estado y política en los orígenes del hospital para mujeres dementes, Buenos Aires, 1852-1870. Buenos Aires: IIEGE-UBA. [Acceso em: Septiembre de 2009]. Disponível em: http://www.unsam.edu.ar/escuelas/politica/ValeriaPita.pdf.

PITA, V. Política, conflictos y consensos en torno al brazo asistencial del estado argentino. La Sociedad de Beneficencia de la Capital, 1880-1910. In: ERASO, Y. Mujeres y asistencia social en Latinoamérica, siglos XIX y XX. Buenos Aires: Ed. Alción, 2009.
RAMACCIOTTI, K. La política sanitaria del peronismo. Buenos Aires: Ed. Biblos, 2009.

ROSANVALLON, $P$. La nueva cuestión social. Buenos Aires: Manantial, 1995.

SAUTÚ, R. La gente sabe. Interpretaciones de la clase media acerca de la libertad, la igualdad, el éxito y la justicia. Buenos Aires: Ediciones Lumiére, 2001.

SIMOIS de BAYÓN, S. Las locas de Buenos Aires (1880-1930). Un acercamiento a las prácticas manicomiales en Argentina. In: MORENO, J. L. (comp). La política social antes de la política social (Caridad, beneficencia y política social en Buenos Aires, siglos XVII a XX). Buenos Aires: Trama editorial, Prometeo libros, 2000.

SPAMPINATO, S. EI tratamiento a la locura en la Argentina desde la Revolución a la constitución del Estado Nacional. Buenos Aires: Monografía Flacso Argentina, 2007.

STAGNARO, J. Evolución y situación actual de la historiografía de la psiquiatría en la Argentina". Rev. Frenia, v.6, 2006.

TESTA, D. La epidemia de poliomielitis en la Ciudad de Buenos Aires. Breve reflexión historiográfica sobre el problema, la cuestión y las políticas sanitarias. Buenos Aires: Monografía Flacso Argentina, 2008. 